\title{
Benchmarking comparison and validation of MCNP photon interaction data
}

\author{
Bethany Colling ${ }^{1, \text { a }}$, I. Kodeli ${ }^{2}$, S. Lilley ${ }^{1}$, and L.W. Packer ${ }^{1}$ \\ ${ }^{1}$ Culham Centre for Fusion Energy, Culham Science Centre, Abingdon, Oxon OX14 3DB, UK \\ 2 Jožef Stefan Institute, Ljubljana, Slovenia
}

\begin{abstract}
The objective of the research was to test available photoatomic data libraries for fusion relevant applications, comparing against experimental and computational neutronics benchmarks. Photon flux and heating was compared using the photon interaction data libraries (mcplib 04p, 05t, 84p and 12p). Suitable benchmark experiments (iron and water) were selected from the SINBAD database and analysed to compare experimental values with MCNP calculations using mcplib $04 p, 84 p$ and $12 p$. In both the computational and experimental comparisons, the majority of results with the $04 p, 84 p$ and $12 p$ photon data libraries were within $1 \sigma$ of the mean MCNP statistical uncertainty. Larger differences were observed when comparing computational results with the 05 t test photon library. The Doppler broadening sampling bug in MCNP5 is shown to be corrected for fusion relevant problems through use of the $84 p$ photon data library. The recommended libraries for fusion neutronics are 84p (or 04p) with MCNP6 and 84p if using MCNP-5.
\end{abstract}

\section{Introduction}

Several photon libraries are available for use with the MCNP-X, MCNP-5 and MCNP-6 code packages [1,2] such as the $04 \mathrm{p}, 84 \mathrm{p}, 05 \mathrm{t}$ and $12 \mathrm{p}$ libraries. Their performance, including the use of the Doppler broadening treatment, was investigated using computational models. A spherical model and an EU fusion demonstration reactor with a helium cooled lithium lead blanket (HCLL DEMO) benchmark model [3] were used.

Photon flux calculated using the $04 p, 84 p$ and $12 p$ data libraries with MCNP-6 was also evaluated for the experimental Photon Leakage Spectra benchmarks [4-6] available in SINBAD [7]. The measurements performed at RFNC-VNIITF, Snezhinsk, using a $14 \mathrm{MeV}$ D-T neutron source placed in the centre of spherical samples of different materials were selected for the comparison exercise.

\section{Photoatomic data libraries}

A number of photon cross-section data libraries are available for use with MCNP for photon transport; 04p, 05t, 84p and 12p. MCNP also has a number of physics options available, including the ability to perform Doppler broadening to incorporate the effect of bound electrons on photon scattering. The $04 \mathrm{p}$ library was considered the standard and default selected library for photon calculations in MCNP.

Since the release of MCNP-5 a bug was found with the use of Doppler broadening [1]. The format for the data provided in $04 \mathrm{p}$ for sampling which atomic shell the electron is bound (provided as a probability density function) does not match the format in which MCNP-5

\footnotetext{
a e-mail: Bethany. Colling@ukaea.uk
}

samples. MCNP-5 samples assuming a cumulative density function. The official report on this MCNP bug is given in [8]. To enable users of MCNP-5 to have corrected data sampling, a temporary library, $84 \mathrm{p}$, was created with the correctly formatted data.

The sampling bug is not evident with MCNP-6 which can recognise the format of the library data and sample accordingly. Therefore using the $04 p$ or $84 p$ photon library data would present the same result; within statistical uncertainty.

A test library, 05t, was developed in order to include form factor data from ENDF/B-VII (rev. 0) as the incoherent and coherent form factors in MCNP-5 and MCNP-X were described as obsolete; affecting photon transport with energies greater than $74 \mathrm{keV}$ [9]. The $05 \mathrm{t}$ data is identical to that in the standard $04 \mathrm{p}$ apart from the inclusion of the updated form factor data. A version of MCNP-X [10] was developed to identify and use the $05 t$ data as it is not compatible with MCNP-5.

The photon library $12 p$ is distributed in a newly developed format that includes photon transport data from $04 p$ and complete relaxation and electron interaction data. The three main developments in this data library are reported [11] to be: (1) the extension of photon transport to lower energies; potentially $1 \mathrm{eV},(2)$ enhanced treatment of atomic relaxation processes, and (3) changes in electron transport methods to allow transport at lower energies [11]. Note that temperature, condensed state, and molecular effects are not yet treated for photons or electrons in MCNP6, and further enhancement of the physics in the transport algorithms may be required for appropriate use of the data in the $\mathrm{eV}$ range. In these calculations only mode $\mathrm{p}$ or $\mathrm{n}, \mathrm{p}$ was used, i.e., only photons or photons and neutrons were considered in the calculations; electron transport was not used. 
Table 1. Libraries and MCNP configurations compared.

\begin{tabular}{|c|c|c|c|c|}
\hline PLIB & MCNP-5 & MCNP-6 & MCNP-X & Incorrect sampling (with \\
\hline 04p & & & & $\begin{array}{l}\text { Dopplen broadening } \\
\text { photon physics) }\end{array}$ \\
\hline $84 p$ & & & & $\begin{array}{l}\text { Correct sampling and } \\
\text { expect similar results }\end{array}$ \\
\hline $05 t$ & & & & $\begin{array}{l}\text { Incompatible } \\
\text { Compared }\end{array}$ \\
\hline $12 p$ & & & & Not compared \\
\hline
\end{tabular}

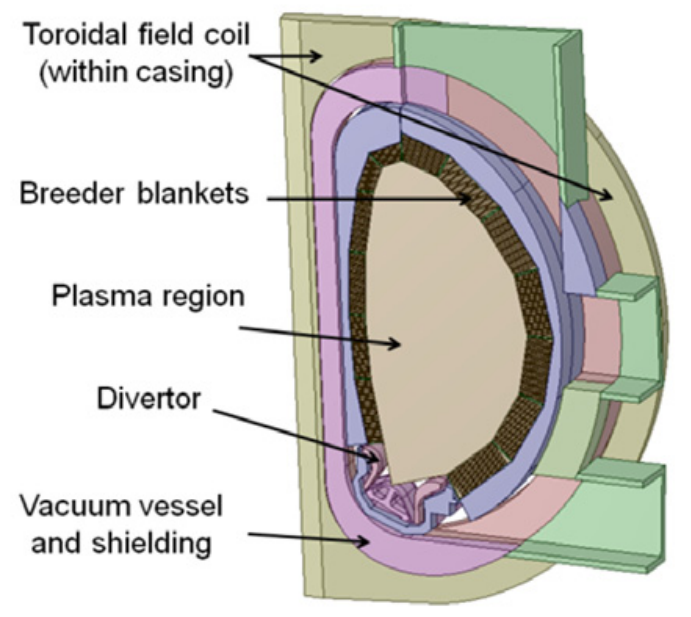

Figure 1. EU HCLL DEMO 2012 benchmark [3] neutronics model.

The photoatomic libraries have been compared using default MCNP-6 physics which includes the use of photon Doppler broadening.

It is important to consider the compatibility of the libraries with different versions of MCNP, namely MCNP-5, MCNP-6 and MCNP-X. A summary of the libraries and MCNP versions compared along with a basic representation of compatibility is given in Table 1 .

\section{Computational benchmark comparisons of photon data libraries}

\subsection{Neutronics modelling and simulations}

A spherical test case was used to compare the effect on the photon flux from the use of different photon libraries. Using a script, the $200 \mathrm{~cm}$ radius spherical shell model was defined with each of the 100 elements featured in the photon data libraries and a separate photon transport calculation performed for each.

The mass density of the material within the sphere has been kept constant at $1 \mathrm{~g} / \mathrm{cm}^{3}$. An $8 \mathrm{MeV}$ photon point source was placed at the centre and the resulting photon flux tallied at 20 spherical surfaces through the material and in 97 energy bins. Each transport calculation was performed without variance reduction with $1 \times 10^{8}$ photon histories.

Further photon library comparisons were made using a fusion relevant model; the benchmark EU HCLL DEMO 2012 model (Fig. 1) [3] with a D-T plasma source definition. Neutron transport was performed using FENDL2.1 [12] neutron cross section data or, where
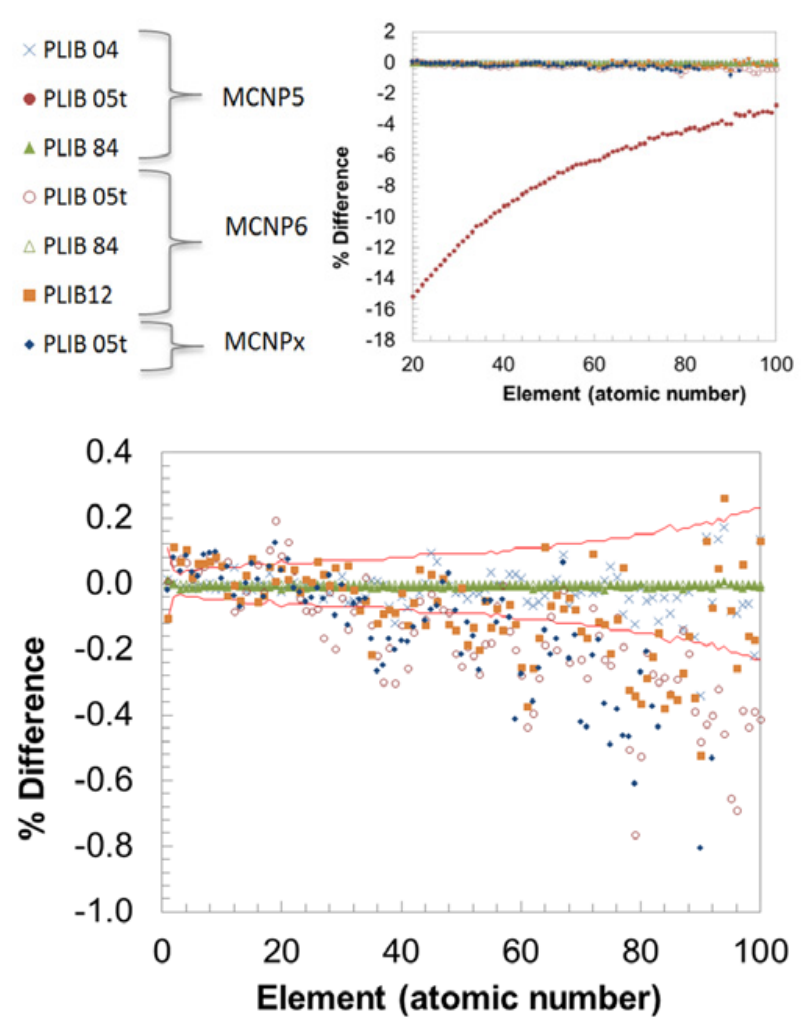

Figure 2. Percentage difference observed from use of different photon libraries with MCNP-5, MCNP-6 and MCNP-X (plot reproduced without $05 t+$ MCNP-5 data and different scale). The red lines denote the $1 \sigma$ statistical uncertainty of the reference case (MCNP-6 + 04p).

unavailable, ENDF-B-VII [13]. Photon and neutron flux and heating have been tallied on a superimposed mesh. Global variance reduction [14] was utilised to establish a weight window map and the simulations performed to $2 \times 10^{9}$ histories.

\subsection{Library comparison results}

The percentage difference observed in the total surface flux (at $160 \mathrm{~cm}$ from the centre source) for each element tested and each MCNP and photon library combination is given in Fig. 2. The percentage difference was calculated by comparing against the reference case results (MCNP$6+04 p)$. The use of MCNP-5 with the 05t library was not expected to be compatible. The plot is therefore reproduced without this data.

The reference case (MCNP-6 + 04p) photon heating through a section of the DEMO model is shown in Fig. 3, normalised to a $2385 \mathrm{MW}$ power plant (i.e., $8.468 \times$ $10^{20}$ neutrons / second). A comparison of the resulting outboard photon heating using different data libraries is also shown.

\subsection{Discussions and conclusions}

The majority of the results from the photon library comparison were as expected (see Table 1). The photon libraries $04 p$ and $84 p$ are identical in cross-section data and differ only in the format of the data provided. This is observed in results from both the spherical test case and the DEMO benchmark. MCNP-6 using 04p gives identical results, within statistical uncertainty, to MCNP-6 using 


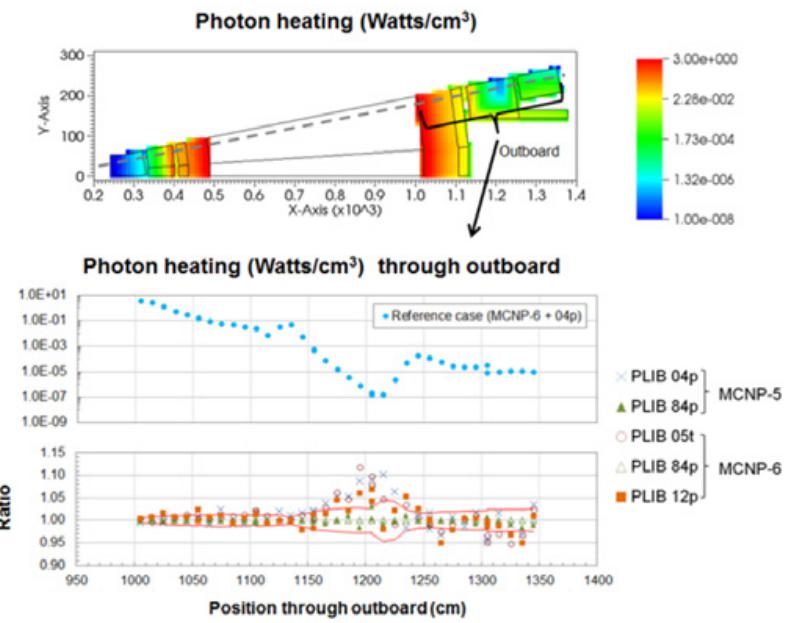

Figure 3. Photon heating and comparison of libraries through outboard section of HCLL DEMO model.

$84 \mathrm{p}$, regardless of whether Doppler broadening is in use or not.

Differences are observed when comparing the use of MCNP-5 with 04p to the reference case with Doppler broadening as expected due to the known bug. However, the majority of results compared were still within 1-2 $\sigma$. Although values within $1 \sigma$ are not strictly identical, one might expect $\sim 68 \%$ agreement. The sampling bug with MCNP-5 is shown to be corrected with the use of $84 \mathrm{p}$.

Use of $05 \mathrm{t}$ with MCNP-5 is shown to provide significantly different results to the reference case; only approximately $18 \%$ of the surface flux tally results were within $1 \sigma$ of the reference case. This is not unexpected as although MCNP-5 will run with the $05 \mathrm{t}$ specified, it is not compatible. It is unclear from the literature whether $05 t$ is compatible with MCNP-6, results presented here show that there is little difference observed in the surface flux when comparing 05t with MCNP-6 and MCNP-X.

The use of $05 \mathrm{t}$ (with MCNP-X) gives up to $11 \%$ difference in the DEMO model photon heating values when compared to the reference case, with approximately $69 \%$ of the voxel values within $1 \sigma$ of the reference case.

The use of $12 p$ gives up to $\sim 7 \%$ difference in the DEMO model photon heating values when compared to the reference case, with approximately $71 \%$ of the voxel values within $1 \sigma$ of the reference case.

\section{Photon transport analysis of RCFC-VNIITF benchmarks from SINBAD}

\subsection{Data libraries and experimental data}

The $04 p, 84 p$ and $12 p$ data libraries were considered in the experimental/computational comparison. Among the shielding benchmark experiments available in SINBAD [7] the two sets of photon leakage spectra benchmarks measured at RFNC-VNIITF (Zababakhin Russian Federal Nuclear Center - All-Russian Scientific Researching Institute of Technical Physics), Snezhinsk [4] were considered to be suitable for the photon validation exercise:

- NEA-1517/74: Photon leakage spectra from Al, Ti, Fe, $\mathrm{Cu}, \mathrm{Zr}, \mathrm{Pb},{ }^{238} \mathrm{U}$ spheres with a central $14 \mathrm{MeV}$ neutron source [5].

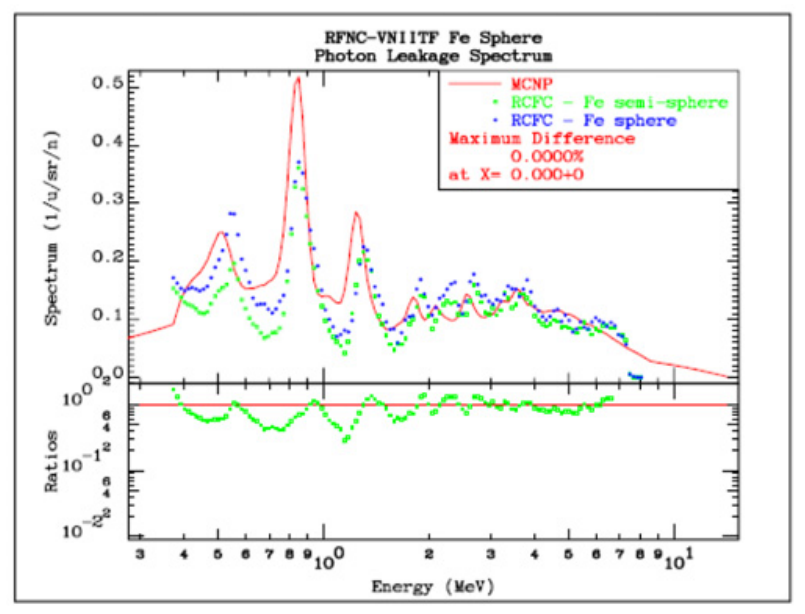

Figure 4. Measured gamma spectra for the RFNC-VNIITF iron sphere measurements compared to the calculated using the $12 p$ photon and ENDF/B-VII.1 neutron library.

- NEA-1517/80: RFNC photon spectra from $\mathrm{H}_{2} \mathrm{O}, \mathrm{SiO}_{2}$ and $\mathrm{NaCl}$ with a central $14 \mathrm{MeV}$ neutron source [6].

\subsection{Benchmark models and experiments}

Both benchmarks used a $14 \mathrm{MeV}$ neutron source placed in the centre of spherical samples of inside diameter $100 \mathrm{~mm}$ and outside diameter $200 \mathrm{~mm}$. The measured photon spectra were normalised to $10^{5} 14 \mathrm{MeV}$ source neutrons. Absolute neutron flux measurements were conducted using the activation method and the reaction ${ }^{27} \mathrm{Al}(\mathrm{n}, \alpha){ }^{24} \mathrm{Na}$. The corresponding measured data are however not available. The attenuation coefficients (total photon leakage from spherical samples per one $14 \mathrm{MeV}$ source neutron) were measured experimentally as a relationship of count rate of the all-wave detector with and without the sample. The method used for the measurements and the neutron-gamma separation is described in $[5,6]$.

The photon spectrum measurements in the energy range $0.3-8.0 \mathrm{MeV}$ were conducted using a scintillation detector having a stilbene crystal with dimensions of $60 \times$ $60 \mathrm{~mm}$. The energy resolution for ${ }^{60} \mathrm{Co}$ lines $(1.17$ and $1.33 \mathrm{MeV}$ ) was about $10 \%$. At energies $>3 \mathrm{MeV}$ it was $6-7 \%$ and at energies $<0.5 \mathrm{MeV}$ it was $15-20 \%$.

The total uncertainty in the measured spectra was estimated at around $12 \%$ composed of the following:

- Statistical uncertainty +-5\%.

- Uncertainty in the detector efficiency: $\pm 5 \%$ within the energy range $0.5-3.0 \mathrm{MeV}$, and $\pm 7-8 \%$ within the range $3-8 \mathrm{MeV}$.

- Uncertainty in mathematical processing of the experimental spectra $\pm 7 \%$.

- Possible uncertainty of both the sphere radii is $\pm 1 \mathrm{~mm}$ and possible uncertainty of target unit dimensions is $\pm 0.1 \mathrm{~mm}$.

Two cases, the iron and water sphere benchmarks, were analysed using MCNP-6 [15]. The neutron cross-sections were taken from the ENDF/B-VII.1 evaluation [13].

\subsection{Results and conclusions}

The results are shown in Fig. 4 and Fig. 5 and indicate good consistency between the measured and calculated spectra. 


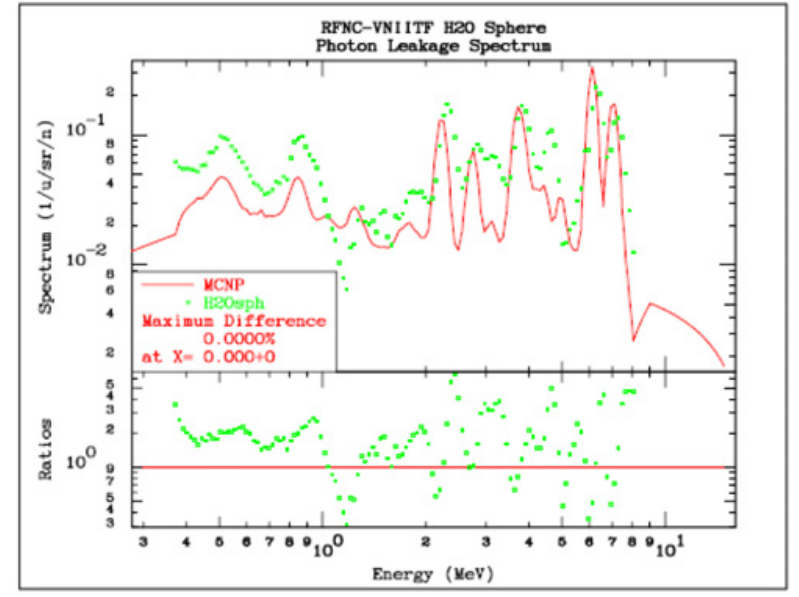

Figure 5. Measured gamma spectra for the RFNC-VNIITF water sphere measurements compared to the calculated using the $12 \mathrm{p}$ photon and ENDF/B-VII.1 neutron library.

The differences observed between the use of the different photon libraries $04 p, 84 p$ and $12 p$ data for the studied cases (iron and water) were small and all within $1 \sigma$ of the MCNP statistical uncertainty.

\section{Summary and future work}

In both the computational and experimental comparisons, the majority of results with the $04 p, 84 p$ and $12 p$ photon data libraries were within $1 \sigma$ of the mean MCNP statistical uncertainty. Larger differences were observed when comparing computational results with the $05 \mathrm{t}$ test photon library. The Doppler broadening sampling bug in MCNP-5 is shown to be corrected through the use of the $84 p$ photon data library.

For fusion neutronics analysis the recommended photon libraries are $84 \mathrm{p} / 04 \mathrm{p}$ with MCNP6 and $84 \mathrm{p}$ if using MCNP-5. In future work the $12 \mathrm{p}$ photon library will be investigated further with regards to fusion neutronics applications.

This work was funded by F4E under contract (F4E-GRT-168.02) and the RCUK Energy Programme [grant number EP/1501045]. The views and opinions expressed herein do not necessarily reflect those of F4E. F4E is not liable for any use that may be made of the information contained herein.

To obtain further information on the data and models underlying this paper please contact PublicationsManager@ccfe. ac.uk.

\section{References}

[1] D. Pelowitz et al., "MCNP6 Users Manual - Code Version 1.0, LA-CP-13-00634 Rev 0"
[2] D. B. Pelowitz (Ed.). "MCNP6 User's Manual", Code version 6.1.1beta, LA-CP-14-00745. Los Alamos National Laboratory (2014)

[3] P. Pereslavtsev, D. Grosse, U. Fischer, et al., "HCLL DEMO 2012 Model - MCNP input." Developed by Forschungszentrum Karlsruhe (KIT) for EUROfusion, 2012

[4] A.I. Saukov, E.N. Lipilina, V.D. Lyutov, "Measurements of Neutron and Photon Leakage from Spherical and Hemispherical Samples with a Central 14-MeV Neutron Source as a Possible Type of Benchmarks", Proc. Int. Conf. Radiation Safety, ICRS10-RPS-2004, May 9-14， 2004， Madeira, Portugal

[5] A.I. Saukov, V.D. Lyutov, E.N. Lipilina, I. Kodeli, "Photon Leakage Spectra from $\mathrm{Al}, \mathrm{Ti}, \mathrm{Fe}, \mathrm{Cu}, \mathrm{Zr}$, Pb, U238 Spheres", OECD/NEA DB Computer Code Package NEA-1517/74 (March 2006)

[6] A.I. Saukov, V.D. Lyutov, E.N. Lipilina, I. Kodeli, "RFNC Photon Spectra from $\mathrm{H} 2 \mathrm{O}, \mathrm{SiO} 2$ and $\mathrm{NaCl}$ ", OECD/NEA DB Computer Code Package NEA1517/80 (May 2007)

[7] I. Kodeli, A. Milocco, P. Ortego, E. Sartori, "20 Years of SINBAD (Shielding Integral Benchmark Archive and Database)", Progress in Nuclear Science and Technology 4, 308-311 (2014)

[8] B.C. Kiedrowski, F.B. Brown, M.C. White, et al., "Testing for the Photon Doppler Broadening Data Sampling" 836

[9] J.S. Hendricks, "Mcnp 0 X Form Factor Upgrade for Improved Photon Transport" 836 (2011)

[10] D.B. Pelowitz, "Mcnpx User' S Manual," LA-CP-1100438, no. April, 2005

[11] H. Hughes, "Quick - Start Guide to Low Energy Photon / Electron Transport in MCNP6," 2013

[12] D. Lopez Al-dama and A. Trkov, "FENDL-2.1: update of an evaluated nuclear data library for fusion applications", IAEA Report INDC(NDS)-46, 2004

[13] M.B. Chadwick et al. ENDF/B-VII.1: Nuclear Data for Science and Technology: Cross Sections, Covariances, Fission Product Yields and Decay Data", Nucl. Data Sheets 112, 2887 (2011)

[14] A. Davis and A. Turner, "Application of Novel Global Variance Reduction Methods to Fusion Radiation Transport Problems", International Conference on Mathematics and Computational Methods Applied to Nuclear Science and Engineering (M\&C 2011)

[15] I. Kodeli, "Photon transport analysis of RCFCVNIITF benchmarks from SINBAD", JEFF/EFF, April 29, 2015, OECD/NEA, Issy-les-Moulineaux 